\author{
Patrícia Ribeiro \\ Fátima Costa \\ Alexandra Monteiro \\ Joana Caldas \\ Madalena Silva \\ Gilda Ferreira \\ Joana Veiga \\ Manuel O. Sousa \\ Maria P. Viegas \\ Ester Santos \\ António J. Gonçalves \\ Aida B. Sousa
}

\section{Polymerase chain reaction screening for fungemia and/or invasive fungal infections in patients with hematologic malignancies}

Received: 4 August 2005

Accepted: 20 October 2005

Published online: 25 January 2006

(C) Springer-Verlag 2006
P. Ribeiro $(\bowtie) \cdot$ F. Costa

A. Monteiro - J. Caldas $\cdot$ M. Silva .

G. Ferreira $\cdot$ J. Veiga $\cdot$ M. O. Sousa ·

A. J. Gonçalves · A. B. Sousa

Department of Hematology,

Hospital dos Capuchos,

Alameda Sto. António dos Capuchos,

1169-050 Lisbon, Portugal

e-mail: patriciamribeiro@sapo.pt

Tel.: +351-21-3136330

Fax: +351-21-3136490

M. P. Viegas · E. Santos

Laboratory of Hematology,

Hospital dos Capuchos,

Alameda Sto António dos Capuchos,

1169-050 Lisbon, Portugal

\author{
Abstract Introduction: Invasive \\ fungal infections (IFIs) are a life- \\ threatening complication in patients \\ with hematologic malignancies, \\ mainly in acute leukemia patients, \\ following chemotherapy. IFI inci- \\ dence is increasing, and associated \\ mortality remains high due to unreli- \\ able diagnosis. Antifungal drugs are \\ often limited by inadequate antimi- \\ crobial spectrum and side effects. \\ Thus, the detection of circulating \\ fungal DNA has been advocated as a \\ rapid, more sensitive diagnostic tool. \\ Patients and methods: Between June \\ 01 and January 03, weekly blood \\ samples $(1,311)$ were screened from \\ 193 patients undergoing intensive \\ myelosuppressive or immunosup- \\ pressive therapy. IFI cases were \\ classified according to European \\ Organization for Research and Treat- \\ ment of Cancer/Mycoses Study
}

\begin{abstract}
Group criteria. Fungal DNA was extracted from whole blood and amplified using polymerase chain reaction (PCR) published primers that bind to the conserved regions of the fungal $18 \mathrm{~S}$ rRNA gene sequence. In our study, two or more consecutive positive samples were always associated with fungal disease. Results: PCR screening predicted the development of IFI to be 17 days (median). This test had a specificity of $91.1 \%$ and a sensitivity of $75 \%$. IFI incidence was $7.8 \%$. Discussion: Therefore, our results confirm the potential usefulness of PCR serial screening and the clinical applicability in everyday routine. PCR screening offers a noninvasive repeatable aid to the diagnosis of IFI.
\end{abstract}

Keywords Neutropenia $\cdot$ Fungal infections $\cdot$ PCR screening

\section{Introduction}

In patients with hematologic malignancies, opportunistic mycosis often develops into severe invasive fungal infection (IFI), which is a life-threatening complication. Uniform experience indicates that this is most frequent in patients with acute leukemia, partly due to the introduction of more intensive combination of chemotherapeutic regimens. These cause more prolonged myelosuppression that results in delays in the recovery of the immune system that will likely end in fatal infections $[1,2]$. Furthermore, these chemotherapy regimens cause mucositis, permitting the establishment of local fungal infections and subsequent hematogenous dissemination. These regimens are delivered through central venous catheters which contribute to the breakdown of normal anatomic barriers, thereby increasing the chance of nosocomial infections [3]. The concomitant use of broad-spectrum antibiotic drugs will suppress the endogenous bacterial flora, permitting fungi overgrowth. Hence, it is not surprising that a gradual increase in IFI incidence has been observed [4]. It is difficult, however, to obtain an accurate estimate of the 
frequency of fungal infections in patients with cancer [5]. Most reports do not provide sufficient information regarding the nature of the underlying disease, many focus on a single fungus, and the frequency of IFI varies considerably between different institutions [6]. To complicate even more the issue, some fungal infections are only discovered at the autopsy [7].

It has become clinical standard practice to administer antifungal therapy empirically to febrile patients [8], making it difficult to determine the proportion of responding patients who were actually infected with fungi. In general, the severity of IFI has led to the adoption of an aggressive approach by most clinical centers, since time is a critical factor in determining successful outcome. However, empirical therapy has risks. Most antifungal drugs have side effects, so early and accurate diagnosis of these infections is important to decrease the use of unnecessary toxic agents [9].

Guidelines have been drawn as to the use of diagnostic techniques to determine more accurately who will need antifungal therapy. These include radiological investigation with computerized tomography scans, invasive procedures for histological examinations and mycological techniques of culture, and finally, serology tests $[10,11]$. However, the performance of invasive procedures is often precluded by profound cytopenias. The detection of fungi by standard culture is often delayed because of slow or absent growth of fungal isolates. Examination of bronchoalveolar lavage may be positive in up to $60 \%$ in cases of aspergillosis with diffuse disease but has limited value for either focal disease or Candida pneumonia [12].

Another diagnostic approach is the use of fungal mannans or galactomannans $[13,14]$. Several techniques, like enzyme immunoassays, radioimmunoassay, and latex particle agglutination tests, have been evaluated for the detection of galactomannan, but their routine use has been difficult due to a low detection limit, to cross-reactivity with exoantigens from bacteria, food mannans, antibiotics, or cytotoxic agents such as cyclophosphamide $[15,16]$. These fungal antigens may even be rapidly removed from circulation by the formation of immune complexes and by receptor-mediated endocytosis by Kupffer cells in the liver, thereby limiting the sensitivity of these diagnostic approaches [17].

The detection of circulating fungal DNA has been considered as a promising tool, more rapid and more sensitive than serologic techniques $[13,18]$. Polymerase chain reaction (PCR) methods are being developed, which may permit early detection by analyzing several different types of samples. Despite the variety of strategies being developed, some have shown limited applicability for routine use in a clinical setting, and so far, most studies were performed in limited numbers of patients [18, 19].

In the present report, a large prospective study was undertaken in a single center to evaluate the value of PCR as a routine screening procedure in patients with hematologic malignancies and to determine the incidence of fungemia and/or IFI.

\section{Patients and methods}

Study population

Between October 2000 and January 2003, 193 patients were admitted to wards equipped with high-efficacy particulate air filters for intensive myelosuppressive or immunosuppressive therapy. These patients (Table 1) were admitted to our institution, and most patients (141) were submitted to the first induction therapy with idarubicin (12 $\mathrm{mg} / \mathrm{m}^{2}$, days $\left.1-3\right)$ plus AraC (100 mg/m², days $\left.1-7\right)$ for all acute myeloid leukemia except promyelocytic acute leukemia patients who are treated with trans-retinoic acid plus idarubicin $\left(12 \mathrm{~g} / \mathrm{m}^{2}\right)$ or for acute lymphoblastic leukemia patients who were treated with alternate cycles of highdose cyclophosphamide, doxorubicin, and AraC and cycles of high-dose methrotexate plus AraC. Consolidation therapy for acute myeloid leukemia was given to 127 patients [mitoxantrone $\left(10 \mathrm{mg} / \mathrm{m}^{2}\right.$, days 2-6) plus high-dose AraC ( $3 \mathrm{~g} / \mathrm{m}^{2}$, days 1,3 , and 5)], 27 patients were on high-dose chemotherapy for autologous peripheral blood progenitor cell transplantation, and finally, 123 patients were on further regimens of chemotherapy [ $=3$ cycles with etoposide (200 mg, days 8-10), mitoxantrone (12 mg, days 1-3), and $\mathrm{AraC}\left(500 \mathrm{mg} / \mathrm{m}^{2}\right.$, days $1-3$ and $\left.\left.8-10\right)\right]$. Severe aplastic anemia patients treated with anti-thymocytic sera and some lymphoma patients treated with ESHAP (etoposide, cis-platinum, doxorubicin, and methylprednisolone) were included in the induction therapy group for analysis simplification.

Fungal infection was classified according to the European Organization for Research and Treatment of Cancer

Table 1 Patient characteristics

\begin{tabular}{ll}
\hline No. Patients & 193 \\
No. of neutropenic episodes & 418 \\
Median age/range (years) & $50 / 16-77$ \\
Male/female & $113 / 80$ \\
Median time to neutrophil recovered (days) & 26 \\
WBC nadir count & $300 / \mathrm{l}$ \\
Diagnosis & \\
Acute myeloid leukemia & 119 \\
Acute lymphoblastic leukemia & 26 \\
Severe aplastic anemia & 8 \\
Non-Hodgkin's and Hodgkin's lymphoma & 40 \\
Treatment episodes & \\
Induction chemotherapy (1st) & 141 \\
Consolidation chemotherapy & 127 \\
Autologous PBSCT & 27 \\
Chemotherapy (relapse patients) & 123 \\
\hline
\end{tabular}


(EORTC)/Invasive Fungal Infections Cooperative Group and Mycoses Study Group (MSG) international consensus criteria [11].

Two peripheral blood samples were taken for microbiology analysis prior to empiric antibiotic institution and whenever a drug modification was necessary. This occurred when unresponsive fever was present or clinical deterioration was observed. Secondary prophylaxis with antifungal therapy was given to patients with a previous episode of proven or probable invasive aspergillosis (IA).

Results from PCR analysis were confidential during the first year of the study and were disclosed after the said period, but clinical workup for disease proceeded as usual.

\section{Study design and PCR assay}

Consecutive blood samples were collected at entry, then weekly until death or hospital discharge, using 5-ml vacutainer tubes with EDTA to minimize chances of environmental contamination. The vacutainer's rubber stopper was carefully removed, and whole blood was aseptically transferred, using a disposable pipette with a filtered tip, into a $12-\mathrm{ml}$ centrifuge tube. Erythrocytes were lysed with $10 \mathrm{ml}$ of erythrocyte lysis buffer $(10 \mathrm{mM}$ Tris $\mathrm{pH} 7.6,5 \mathrm{mM}$ $\mathrm{MgCl}_{2}, 10 \mathrm{mM} \mathrm{NaCl}$ ) by incubation for $10 \mathrm{~min}$ at $37^{\circ} \mathrm{C}$ and then separated by centrifugation ( $5 \mathrm{~min}, 1,800 \mathrm{rpm}$ ). The procedure was repeated twice. The pellet was then lysed by incubation (water bath $65^{\circ} \mathrm{C}, 45 \mathrm{~min}$ ) with a leukocyte lysis buffer (10 mM Tris $\mathrm{pH}$ 7.6, $10 \mathrm{mM}$ EDTA $\mathrm{pH} 8.0,50 \mathrm{mM} \mathrm{NaCl}, 0.2 \%$ sodium dodecyl sulfate, $200 \mu \mathrm{g} / \mathrm{ml}$ proteinase $\mathrm{K}$ ), then separated by centrifugation $(10 \mathrm{~min}, 4,000 \mathrm{rpm})$ [20]. $\mathrm{NaOH}(1 \mathrm{ml}$ of $50 \mathrm{mM})$ was added, followed by incubation for $10 \mathrm{~min}$ at $95^{\circ} \mathrm{C}$. Then, $1 \mathrm{ml}$ of $1 \mathrm{mM}$ Tris $\mathrm{pH} 7.0$ was added for neutralization. Fungal cell walls were lysed by adding a digestion mixture of $50 \mathrm{mM}$ Tris $\mathrm{pH} 7.5,1 \mathrm{mM}$ EDTA, beta-2mercaptoethanol $28 \mathrm{mM}, 300 \mu \mathrm{g} / \mathrm{ml}$ lyticase solution (Sigma) [20]. Incubation (room temperature, $45 \mathrm{~min}$ ) was followed by centrifugation for $10 \mathrm{~min}$ at $5,000 \mathrm{rpm}$. Supernatant was carefully removed, the pellet was resuspended into DNAzol BD reagent (Gibco BRL), and extraction was performed according to manufacturer's recommendations.

Fungal DNA $(5 \mu \mathrm{l})$ was amplified in a $50-\mu$ l reaction volume, with 1.25 U Taq polymerase (Gibco BRL) and with panfungal PCR published primers [21] that bind to the conserved regions of the fungal $18 \mathrm{~S}$ rRNA gene sequence. PCR-amplified products of $197 \mathrm{bp}$ were obtained by using the forward $5^{\prime}$-ATTGGAGGGCAAGTCTGGTG-3' and the reverse 5'-CCGATCCCTAGTCGGCATAG-3' primers (synthesized by Gibco BRL). Amplification started with a denaturation step at $94^{\circ} \mathrm{C}$ for $5 \mathrm{~min}, 35$ cycles of $94^{\circ} \mathrm{C}$ for $45 \mathrm{~s}, 62^{\circ} \mathrm{C}$ for $1 \mathrm{~min}$ and $30 \mathrm{~s}, 72^{\circ} \mathrm{C}$ for $2 \mathrm{~min}$, ending in a final elongation step at $72^{\circ} \mathrm{C}$ for $10 \mathrm{~min}$. Established PCR negative and positive control samples were used in every assay. PCR final products were electrophoresed through a $1.8 \%$ agarose gel stained with ethidium bromide and UV photographed (Kodak 120 camera system).

This study also allows for indirect characterization of fungal species. Using the primers described above, occasionally, a 492- and 503-bp PCR-amplified products were obtained due to the highly variable internal transcribed spacer (ITS) regions, common in the general fungus genetic code. Looking through the fungal nucleotide published sequences in the GenBank database (accession numbers AJ005123 and AF320009), we found that these could match Candida albicans and Ajellomyces capsulatus (Histoplasma capsulatus), respectively.

Statistical analysis

The calculation of sensitivity of the PCR assay was based on the episodes with proven IA, probable IA, candidemia, and chronic disseminated candidiasis (CDC). For the calculation of specificity, episodes without criteria for IFI according to the EORTC/MSG 2002 international consensus classification [11] were considered true negative episodes.

\section{Results}

A total of 1,311 whole blood samples from 418 neutropenic episodes (mean 3.14 samples/episode, mean 6.8 samples per patient, range 1-9) were analyzed by PCR: 153 episodes were classified as fever with microbiological documentation (143 with bacteremia; 2, viral infection; 1, histoplasmosis; 2, Pneumocystis carinii; 2, tuberculosis; and 3, candidemias: 2 C. albicans and 1 Candida krusei), 75 episodes were characterized as fever of unknown origin, 39 episodes without fever, and 151 episodes had fever with clinical documentation among which, 60 were pneumonias. Details of 51 patients suspected of having pulmonary IA are shown in Table 2, paired with PCR results and the number of samples tested. The remaining correspondence of sampling and PCR positive assays are in Table 3. In the group characterized by fever with clinical documentation, a correspondence between some episodes and patients was observed: four episodes of CDC (corresponding to four

Table 2 Blood samples tested by PCR in different patients

\begin{tabular}{lccr}
\hline $\begin{array}{l}\text { EORTC/MSG } \\
\text { criteria IA }\end{array}$ & No. of patients & $\begin{array}{l}\text { PCR positive } \\
\text { once (twice) }\end{array}$ & Tested samples \\
\hline Proven & 1 & $1(0)$ & 1 \\
Probable & 11 & $11(4)$ & 61 \\
Possible & 19 & $5(1)$ & 111 \\
Unlikely & 20 & $2(0)$ & 83 \\
\hline
\end{tabular}


Table 3 PCR tests and classification episodes

\begin{tabular}{llll}
\hline Classification & No. of episodes & PCR positive tests & Tested samples \\
\hline Fever (unknown origin) & 75 & $10(5 / 1$ patient $)$ & 210 \\
Episodes without fever & 39 & 1 & 80 \\
Clinical documentation fever & 151 & 22 & 602 \\
& $(4 \mathrm{CDC})$ & $(6)$ & $(25)$ \\
Microbiological documentation fever & 153 & 24 & 419 \\
& $(3$ Candidem) & $(4)$ & $(5)$ \\
& $(1$ Histoplasm) & $(8)$ & $(30)$ \\
\hline
\end{tabular}

patients, one patient had a candidemia and, during another neutropenic episode, developed a CDC).

On the whole 1,311 samples, 76 samples were PCR positive. Only 15 patients fulfill all the requirements, according to the EORTC/MSG criteria, for having a deep fungal disease (Table 4). The IFI incidence among the 193 patients is $7.8 \%$. Two or more consecutive or intermittently positive PCR samples for the same patient were observed for several patients, and, in these cases, the positivity was always associated with fungal disease. Screening predicted the appearance of IFI to be 17 days (median). In one case, a patient with relapsed acute lymphoblastic leukemia had intermittently positive PCR samples, but a fungal infection was not found. She was discharged and readmitted one week later with fever, PCR positive tests, and a candidemia. From the first PCR positive test until microbiological determination, a 4-week period elapsed.

The evaluation of the PCR screening impact is shown in Table 4 . We found $7.2 \%$ of false positives and $2.5 \%$ of false negatives. In our study, a specificity of $91.1 \%$ and a sensitivity of $75 \%$ were obtained.

\section{Discussion}

In the present study, we found an IFI incidence of $7.8 \%$ in patients with hematologic malignancies. The criteria used in this study were based on the definitions proposed by the EORTC/MSG consensus [11], which may lead to an underestimate of the incidence of IFI infections. However, candidemia rates of $6.3 \%[22]$ and aspergillosis rates of $6 \%$ [23] were found in previous reports at our institution, and recently, similar rates have been published [24]. A substantial number of studies have been focusing on the rising of IFI incidence in the last decades [3, 4, 11, 18]; there is, however, a considerable variation in the range of

Table 4 Data comparing PCR tests and final diagnosis according to EORTC/MSG

\begin{tabular}{lccc}
\hline PCR & With IFI & Without IFI & Total patients \\
\hline Positive & 15 & 14 & 29 \\
Negative & 5 & 159 & 164 \\
Total patients & 20 & 173 & 193 \\
\hline
\end{tabular}

organisms isolated in different hospitals, and unfortunately, it is difficult to have precise numbers $[3,5]$. Probably, on the whole, IFI incidence has stabilized; between 1950 and 1980 , some studies reported a variable incidence of 20 to $60 \%$ among patients with acute leukemia [7, 25].

This report describes a panfungal PCR assay based on a lysis centrifugation method, which allows for repeated testing on multiple occasions. Although the procedure is easy to perform, the sensitivity of the assay was quite low (75\%) compared to some published data $[19,26]$. However, in these reports, only IA was screened. Our results are in accordance with others $[18,21]$, but specimen preparation can have a significant impact on the sensitivity. DNA isolation from some fungal organisms is difficult due to cell walls that are not readily susceptible to lysis, although the presence of the usual smear of DNA on the agarose gel permits differentiation from other causes of false negative tests. Other reasons for a false negative test are the presence of potential inhibitors of the fungus PCR reaction and/or the lower amount of fungal DNA in the sample [20]. Antifungal treatment and hepatic clearance may cause intermittent clearing of the organism from the bloodstream [17]. PCR negativity may also be due to the half-life of circulating DNA [27]. The use of universal probes designed to detect a single-copy gene has low sensitivity when compared to specific probes targeting multicopy genes [28], but these are time consuming and not applicable in everyday routine, considering the large number of potentially pathogenic fungi. Furthermore, the chosen sequences in our study have highly variable ITS regions which allow for exploitation for species identification and further confirmation by sequencing analysis. On the other hand, since fungi are ubiquitous in the environment, panfungal PCR methods are more likely to yield contaminated products, while the use of specific probes will reduce this possibility [13, 20]. Sensitivity will probably improve if amplification is achieved by nested PCR [13, 19], although some reports have showed similar sensitivity results [26] to ours. Sensitivity will also improve by using real-time PCR [26]. This latter method reduces the risk of contamination and permits fungal quantification. This will answer some clinical questions as the correlation between the disappearance of fungal DNA from the blood and treatment success; in fact, it may even be used to tailor treatment duration. Recently, a quantitative PCR assay with the 
LightCycler detection system was described as promising [26].

The specificity in our study was $91.1 \%$, which is quite high and in concordance with other studies [18, 19, 26]. The discrepancy encountered in our study regarding sensitivity and specificity may be explained by a bias introduced by a higher sampling frequency in patients with fever and clinical documentation, namely, possible IA and unlikely IA compared to proven IFI (Tables 2 and 3). False positive results from 8 to $38 \%$ have been observed [13, 27]. Some patients were intermittently positive in our study, which may be related to exogenous contamination. Therefore, any positive result must be confirmed with a second positive result taken on a different occasion. Furthermore, fungemia may be intermittent due to hepatic clearance [17].

The present study describes a universal fungal detection protocol that is simple, rapid, repeatable, and noninvasive and has clinical applicability in everyday routine. As a screening method, it is feasible and can be applied to all sorts of samples [whole blood as demonstrated but also to British antilewisite and histology material (unpublished data)]. It allows for early diagnosis provided regular serial sampling is obtained. It is cost-effective when compared to the economical impact of administering antifungal drugs to all patients. Sensitivity has to be improved by introducing better amplification methods as discussed. Our results suggest that molecular biology methods are more sensitive than the standard sera testing by immunological/enzymelinked immunosorbent assay methods [13, 27]. The advantage of a future commercial PCR diagnostic kit would be to standardize methodology between centers and reduce the possibility of contamination. Although PCR-based tests cannot replace other diagnostic procedures, they represent a valuable aid. A high index of suspicion and careful clinical and radiological examinations are requisites to early identification of IFI patients and aggressive treatment steps, which are mandatory for outcome success in patients with hematologic malignancies. However, PCR may lead to preemptive antifungal therapy, may monitor therapeutic response, may detect drug resistant species, and will slowly modify the severity of fungal infections. We also suggest a multicenter trial to validate our results, although we favor a more sensitive approach as a quantitative PCR method.

Acknowledgement The authors are grateful to Lilly Pharma for providing the molecular biology equipment that made this study possible.

\section{References}

1. Meunier F, Aoun M, Bitar N (1992) Fungal infections in immunocompromised hosts. Clin Infect Dis 14:S120 S125

2. Uzun O, Anaissie EJ (1996) Problems and controversies in the management of hematogenous candidiasis. Clin Infect Dis 22:S95-S101

3. Pfaller MA (1996) Nosocomial candidiasis: emerging species, reservoirs and modes of transmission. Clin Infect Dis 22:S89-S94

4. Richardson MD, Kokki MH (1998) Antifungal therapy in bone marrow failure. Br J Haematol 100:619-628

5. Denning DW (1996) Therapeutic outcome in invasive aspergillosis. Clin Infect Dis 23:608-615

6. Bodey GP, Vartivarian S (1989) Aspergillosis. Eur J Clin Microbiol Infect Dis 8:413-437

7. Bodey G, Bueltmann B, Duguid W, Gibbs D, Hanak H, Hotchi M, Mall G, Martino P, Meunier F, Milliken S, Naoe S, Okudaira M, Scevola D, van't Wout J (1992) Fungal infections in cancer patients: an international autopsy survey. Eur J Clin Microbiol Infect Dis 11:99-109
8. Pizzo PA (1993) Managemant of fever in patients with cancer and treatmentinduced neutropenia. N Engl J Med 328:1323-1332

9. Walsh TJ, Finberg RW, Arndt C et al for the National Institute of Allergy and Infectious Diseases Mycoses Study Group (1999) Liposomal amphotericin $\mathrm{B}$ for empirical therapy in patients with persistent fever and neutropenia. N Engl J Med 340:764-771

10. Pappas PG, Rex JH, Sobel JD, Filler SG, Dismukes WE, Walsh TJ, Edwards JE (2004) Guidelines for treatment of candidiasis. Clin Infect Dis 38:161-189

11. Ascioglu S, Rex JH, de Pauw B et al for the Invasive Fungal Infections Cooperative Group of the European Organization for Research and Treatment of Cancer and Mycoses Study Group of the National Institute of Allergy and Infectious Diseases (2002) Defining opportunistic invasive fungal infections in immunocompromised patients with cancer and hematopoietic stem cell transplants: an international consensus. Clin Infect Dis 34:7-14

12. Yu VL, Muder RR, Poorsattar A (1986) Significance of isolation of aspergillus from the respiratory tract in diagnosis of invasive pulmonary aspergillosis. Am J Med 81:249-254
13. Yeo SF, Wong B (2002) Current status of nonculture methods for diagnosis of invasive fungal infections. Clin Microbiol Rev 15:465-484

14. Latgé J-P (1999) Aspergillus fumigatus and aspergillosis. Clin Microbiol Rev $12: 310-350$

15. Adam O, Aupérin A, Wilquin F, Bourhis J-H, Gachot B, Chachaty E (2004) Treatment with piperacillintazobactam and false-positive aspergillus galactomannan antigen test results for patients with hematological malignancies. Clin Infect Dis 38:917-920

16. Hashiguchi K, Niki Y, Soejima R (1994) Cyclophosphamide induces false-positive results in detection of aspergillus antigen in urine. Chest 105:975-976

17. de Repentigny L (1992) Serodiagnosis of candidiasis, aspergillosis, and cryptococcosis. Clin Infect Dis 14:S11-S22

18. Lass-Flori C, Aigner J, Gunsilius E, Petzer A, Nachbaur D, Gastl G, Einsele H, Löffler J, Dierich MP, Würzner R (2001) Screening for Aspergillus spp. Using polymerase chain reaction of whole blood samples from patients with haematological malignancies. Br J Haematol 113:180-184 
19. Williamson ECM, Leeming JP, Palmer HM, Steward CG, Warnock D, Marks DI, Millar MR (2000) Diagnosis of invasive aspergillosis in bone marrow transplant recipients by polymerase chain reaction. Br J Haematol 108:132 139

20. Van Burik J-A, Myerson D, Schrekhise RW, Bowden RA (1998) Panfungal PCR assay for detection of fungal infection in human blood specimens. J Clin Microbiol 36:1169-1175

21. Einsele H, Hebart H, Roller G, Löffler J, Rothenhofer I, Müller CA, Bowden RA, van Burik J, Engelhard D, Kanz L, Schumacher U (1997) Detection and identification of fungal pathogens in blood by using molecular probes. J Clin Microbiol 35:1353-1360

22. Ribeiro P, Sousa AB, Nunes O, Aveiro F, Fernandes JP, Gouveia J (1997) Candidemia in acute leukemia patients. Support Care Cancer 5:249-251
23. Ferreira G, Cruz S, Fernandes J, Sousa AB, Gouveia J (1996) Aspergilose invasiva em doentes com leucemia aguda. New Trends in Hemato-oncology II, Porto (abstract)

24. Menichetti F (2004) How to improve the design of trials of antifungal prophylaxis among neutropenic adults with acute leukemia. Clin Infect Dis 39: S181-S184

25. Bodey GP (1966) Fungal infections complicating acute leukemia. J Chronic Dis 19:667-687

26. Buchheidt D, Hummel M, Schleiermacher D, Spiess B, Schwerdtfeger R, Cornely OA, Wilhelm S, Reuter S, Kern W, Südhoff T, Handan M, Hehlmann (2004) Prospective clinical evaluation of a lightcycler-mediated polymerase chain reaction assay, a nested-PCR assay and a galactomannan enzyme-linked immunosorbent assay for detection of invasive aspergillosis in neutropenic cancer patients and haematological stem cell transplant recipients. Br J Haematol 125:196-202
27. Bretagne S, Costa JM, Bart-Delabesse E, Dhedin N, Rieux C, Cordonnier C (1998) Comparison of serum galactomannan antigen detection and competitive polymerase chain reaction for diagnosing invasive aspergillosis. Clin Infect Dis 26:1407-1412

28. Sandhu GS, Kline BC, Stockman L, Roberts GD (1995) Molecular probes for diagnosis of fungal infections. J Clin Microbiol 33:2913-2919 